2001. We provided care for 77 casualities; among them, 22 required analgesia that was performed using the 50 -to-50\% mixture of oxygen and nitrous oxide.

Keywords: Center of Disaster and Emergency Medicine; disasters; emergencies; nitrous oxide; oxygen; pain; relief

Prebosp Disast Med 2002;17(s2):s80-s81.

\section{Disaster and Emergency Medicine at Nursing \\ Facilities in Japan}

Yoshio Murayama, MD; ${ }^{1}$ Makoto Aono, $M D ;^{2}$ Yoshibiro

Yagisbita, MD; ${ }^{3}$ Yukio Shimizu, $M D^{4}$

1. Kobe National Hospital, Japan

2. Teishinkai Hospital, Japan

3. International Medical Center of Japan, Japan

4. Esan Hospital, Japan

Introduction: Japan is rapidly becoming one of the most aged societies in the world. In preparation for such an aged society, a long-term-care insurance law has been established, and many nursing facilities are being built.

Methods: In order to investigate the situation of emergency medicine in nursing facilities for the elderly, a questionnaire survey was conducted. Because Japan has an environment prone to disaster, a questionnaire survey to nursing facilities in Kobe City was also conducted to investigate the effects of the Great Hanshin Earthquake in 1995.

Results: The percentage of residents transported to hospitals by ambulance at nursing facilities was approximately $1.8 \%$ per year. Among such patients who were transported to hospitals by ambulance, some seemed to have poor prognosis because of their declining physical functions due to senescence. However, there were some residents who had the possibility of a relatively good prognosis if appropriate treatment was applied, including those who had choked on food and suffered trauma. There were only a few nursing facilities that received severe damage from the earthquake, even in the highly stricken area.

Conclusion: This presentation outlines the present situation of emergency medicine and the damage from the earthquake disaster at nursing homes in Japan.

Keywords: aging; elderly; disaster; earthquakes; emergency medicine; nursing homes; prognosis

Prehosp Disast Med 2002;17(s2):s81.

\section{Quality of Emergency Trauma Care in India: An Analysis Based on TRISS Methodology in Mumbai University Hospital \\ V. Murlidbar; NandiniJ. Desa; Nobbojit Roy \\ BARC Hospital, Bombay, India}

Introduction: The level of care to trauma victims in the emergency setting cannot be compared easily among trauma centers around the world. In this prospective study, the trauma injury severity score (TRISS) methodology is used to compare the trauma care offered at a metropolitan university hospital of a developing country to the standardized Major Trauma Outcome Study (MTOS) in the United States.

Methods: Between 01 August 2001 and 31 May 2002, 1,074 severely injured patients admitted to the emergency ward were included in the study. Survival analysis was completed for $98.3 \%$ of the patients.
Results: The majority of the patients were men (84\%) and the average age was 31 years. Of these patients, $90.4 \%$ suffered from blunt injuries, the most common resulting from road traffic accidents $(39.2 \%)$. The predicted mortality rate was $10.9 \%$, while the observed mortality rate was $21.3 \%$. The mean revised trauma score was $6.6 \pm 1.65$, and the mean Injury Severity Score (ISS) was $16.7 \pm 10.67$. The average probability of survival ( $\mathrm{Ps}$ ) was 89.14 . The $\mathrm{M}$ - and $Z$-statistics were 0.84 and -14.1593 , respectively.

Conclusion: Those persons who were injured in India often were older. When compared with the MTOS, the injuries were more severe and resulted in poorer outcomes. Other factors that influenced outcomes were the lack of prehospital care and injury prevention strategies, availability of informal careers, premorbid nutritional status, and economic constraints of the healthcare system. In a developing country like India, economic and institution-bound factors, in addition to the specific limitations of the TRISS methodology, were responsible for the differences between predicted and observed mortality.

Keywords: comparison; India; injury severity score (ISS); Major Trauma Outcome Study (MTOS); mortality rate; road traffic accidents; severity scores; trauma; trauma injury severity score (TRISS)

Prebosp Disast Med 2002;17(s2):s81.

Transportation of Neonates with Congenital Heart

Disease: Appropriate Respiratory Management

S. Nakano; ${ }^{1}$ R.N. Justo; ${ }^{1}$ Y. Koido, ${ }^{2}$ Y. Yamamoto ${ }^{2}$

1. Department of Cardiology, The Prince Charles Hospital, Brisbane, Australia

2. Department of Emergency and Critical Care Medicine, Nippon Medical School, Tokyo, Japan

Background: Attention should be paid in some cardiac conditions in which excessive oxygen dilates pulmonary vessels, leading to congestive heart failure (CHF) or constricts a patent ductus arteriosus (PDA), leading to cyanosis or insufficient systemic circulation.

Objective: To report neonatal cardiac disease requiring transport with respiratory management.

Method: Eighty neonates (0-28 days of age) transported between 2001 and 2002 to the tertiary hospitals in Brisbane for cardiac intensive care and/or emergency surgery were categorized into one of three groups, based on desirable respiratory management:

A. Sufficient oxygen (e.g., persistent pulmonary hypertension);

B Minimal oxygen (e.g., transposition of great arteries, coarctation of aorta, total anomalous pulmonary venous drainage); or

C. Minimal oxygen with controlled ventilation (e.g., hypoplastic left heart syndrome).

Inappropriate respiratory management was defined when neonates without desirable respiratory management on transport developed symptoms such as acidosis or CHF. Results: Group A contributed $1.3 \%$ of total, $B=83.8 \%$, and $C=15.0 \%$. Inappropriate respiratory management was observed in some neonates in Groups $\mathrm{B}$ and $\mathrm{C}(\mathrm{C}>\mathrm{B})$.

Conclusions: Most of the neonates with cardiac disease requiring transport should be given minimal oxygen. The group including hypoplastic left heart syndrome in which 